\title{
Expression and Roles of Teneurins in Zebrafish
}

\section{OPEN ACCESS}

Edited by:

Antony Jr. Boucard,

Centro de Investigación y de Estudios Avanzados (CINVESTAV), Mexico

Reviewed by:

Timothy Mosca,

Thomas Jefferson University,

United States

Elena Seiradake,

University of Oxford, United Kingdom

*Correspondence:

Robert Hindges

robert.hindges@kcl.ac.uk

t These authors have contributed

equally to this work

$¥$ Present address:

Katherine E. Trevers,

Department of Cell

and Developmental Biology, University

College London, London,

United Kingdom

Paride Antinucci,

Department of Neuroscience,

Physiology and Pharmacology,

University College London, London, United Kingdom

Specialty section:

This article was submitted to Neuroendocrine Science,

a section of the journal

Frontiers in Neuroscience

Received: 24 October 2018

Accepted: 12 February 2019

Published: 12 March 2019

Citation:

Cheung A, Trevers KE

Reyes-Corral M, Antinucci $P$ and

Hindges $R$ (2019) Expression

and Roles of Teneurins in Zebrafish.

Front. Neurosci. 13:158.

doi: 10.3389/fnins.2019.00158

\section{Angela Cheung ${ }^{1,2 t}$, Katherine E. Trevers ${ }^{1,2 t \neq}$, Marta Reyes-Corral ${ }^{1}$, Paride Antinucci ${ }^{1 \neq}$ and Robert Hindges ${ }^{1,2 *}$}

${ }^{1}$ Centre for Developmental Neurobiology, King's College London, London, United Kingdom, ${ }^{2}$ MRC Centre

for Neurodevelopmental Disorders, King's College London, London, United Kingdom

The teneurins, also known as Ten-m/Odz, are highly conserved type II transmembrane glycoproteins widely expressed throughout the nervous system. Functioning as dimers, these large cell-surface adhesion proteins play a key role in regulating neurodevelopmental processes such as axon targeting, synaptogenesis and neuronal wiring. Synaptic specificity is driven by molecular interactions, which can occur either in a trans-homophilic manner between teneurins or through a trans-heterophilic interaction across the synaptic cleft between teneurins and other cell-adhesion molecules, such as latrophilins. The significance of teneurins interactions during development is reflected in the widespread expression pattern of the four existing paralogs across interconnected regions of the nervous system, which we demonstrate here via in situ hybridization and the generation of transgenic BAC reporter lines in zebrafish. Focusing on the visual system, we will also highlight the recent developments that have been made in furthering our understanding of teneurin interactions and their functionality, including the instructive role of teneurin-3 in specifying the functional wiring of distinct amacrine and retinal ganglion cells in the vertebrate visual system underlying a particular functionality. Based on the distinct expression pattern of all teneurins in different retinal cells, it is conceivable that the combination of different teneurins is crucial for the generation of discrete visual circuits. Finally, mutations in all four human teneurin genes have been linked to several types of neurodevelopmental disorders. The opportunity therefore arises that findings about the roles of zebrafish teneurins or their orthologs in other species shed light on the molecular mechanisms in the etiology of such human disorders.

Keywords: teneurin/Odz, retinal ganglion cell, amacrine cell, visual system, synapse adhesion molecule, zebrafish

\section{INTRODUCTION}

As one of the most complex systems in nature, the functionality of the nervous system is highly dependent on the formation of precise synaptic connections between neurons during development. While progress is still being made in furthering our understanding of these mechanisms, it is becoming increasingly evident that synaptic specificity is a finely attuned process involving a plethora of cell adhesion molecules that act in a combinatorial manner to generate diverse cellular interactions. The teneurins, also known as Ten-m/Odz, are one family of such cell adhesion molecules that has been implicated, among others, in regulating the specificity of synaptic connections.

A phylogenetically conserved family of type II transmembrane glycoproteins first discovered in the early 1990s in Drosophila, the teneurins have been shown to be involved in intercellular signaling during development (Tucker and Chiquet-Ehrismann, 2006; Tucker et al., 2012). Their key role in mediating basic neurodevelopmental processes such as axon guidance and synaptic 
partner matching (Drabikowski et al., 2005; Hong et al., 2012) is reflected in the high expression of teneurins in the central nervous system. Across species, $C$. elegans has a single teneurin (Ten-1), Drosophila have two (Ten-m and Ten-a) and all vertebrates have four paralogs (tenm1-4). Sequence similarity is high between paralogs, with human teneurin paralogs sharing 58-70\% sequence identity (Jackson et al., 2018).

The teneurins themselves are large proteins of around $300 \mathrm{kDa}$ with a smaller $\mathrm{N}$-terminal intracellular domain, a single span transmembrane domain and large C-terminal extracellular region (Rubin et al., 1999; Tucker and Chiquet-Ehrismann, 2006; Figure 1). While the intracellular domain is able to interact with the cytoskeleton, as has been shown with tenm 1 (Nunes et al., 2005), the highly conserved $200 \mathrm{kDa}$ extracellular domain of teneurin, which contains eight epidermal growth factor (EGF)-like repeats and five NHL (NCL-1, HT2A, and Lin-41) repeats, dimerizes in cis to mediate both homo- and heterophilic interactions (Beckmann et al., 2013). In addition, the teneurins can interact trans-synaptically with other cell adhesion molecules such as latrophilins via their NHL domains (Boucard et al., 2014). Tenm2, for example, interacts across the synaptic cleft with presynaptic latrophilin1 to mediate calcium signaling and synapse formation (Vysokov et al., 2016). Recent X-ray crystallography and cryo-EM imaging

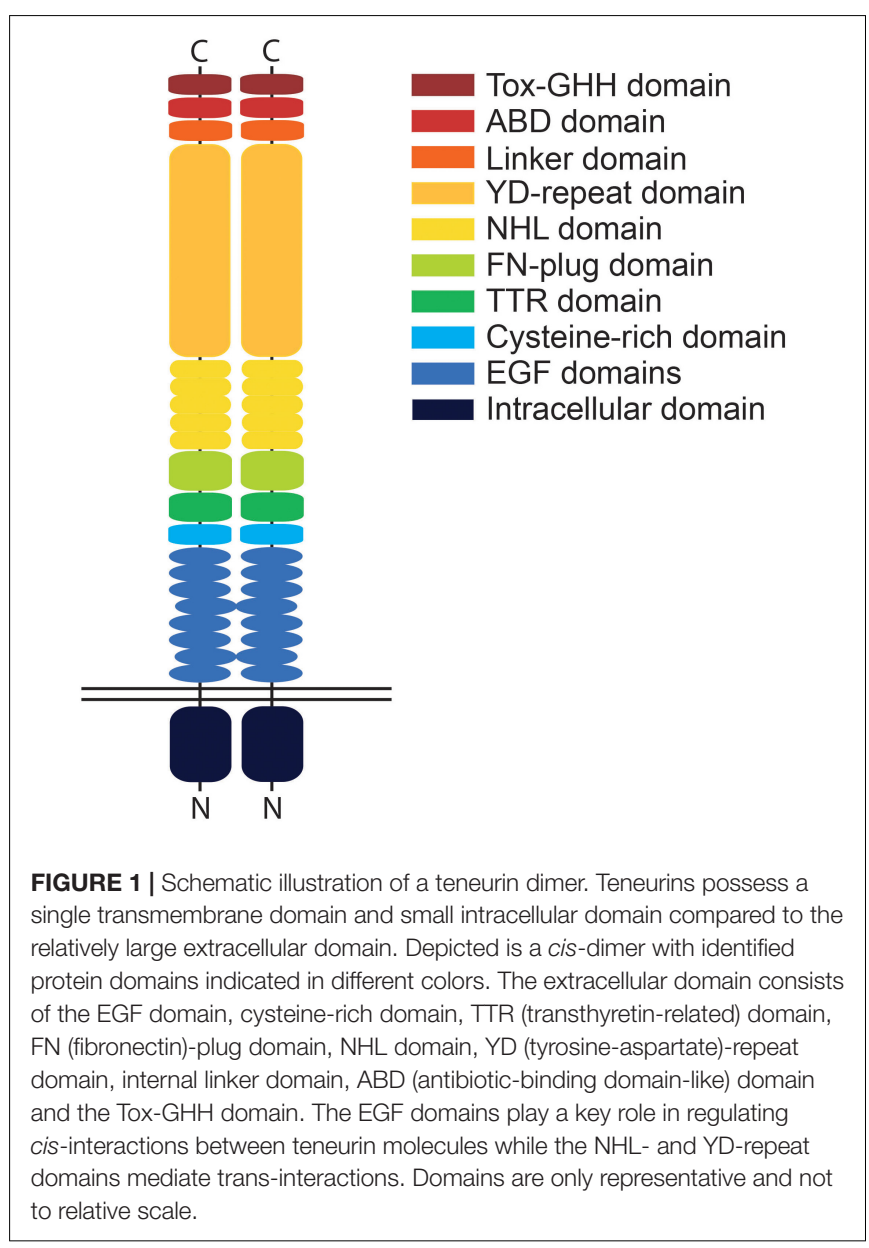

data has demonstrated that the ectodomains of tenm 2 and 3 exist in a large $\beta$-barrel conformation consisting of an eight sub-domain super-fold structured on a spiraling YD-repeat shell domain (Jackson et al., 2018; Li et al., 2018). Further elucidation of the structural interactions between teneurins and other proteins would provide insight into functionality and factors driving the molecular diversity underpinning synaptic connectivity or even highlight possible new interaction partners.

Indeed, the importance of teneurins in regulating synaptic partner matching and functional connectivity is well demonstrated in the vertebrate visual system where retinal ganglion cells (RGCs) form specific connections with their synaptic partners. In zebrafish, tenm 3 is required by RGCs and amacrine cells for acquiring correct structural and functional connectivity in vivo, with tenm 3 knockdown or knockout leading to defects in RGC and amacrine cell dendrite stratification in the retina and the disrupted development of orientation selectivity (Antinucci et al., 2013, 2016). Tenm3 has also recently been shown to regulate topographic circuit assembly in the hippocampus of mice (Berns et al., 2018). More broadly, the teneurins are strongly implicated in the establishment of visual mapping in mice (Leamey and Sawatari, 2014). Anterograde tracing of RGC axons in tenm 2 and tenm 3 knockout mice showed notable aberrant changes in the mapping of ipsilateral projections from the retina to the dorsal lateral geniculate nucleus (dLGN) and the superior colliculus (Leamey et al., 2007; Young et al., 2013).

Although the fundamental role of teneurins in establishing synaptic connectivity is becoming increasingly apparent, data on the spatio-temporal expression pattern of teneurins across the central nervous system, and its functional and physiological significance, is largely lacking. Through in situ hybridization and the generation of transgenic bacterial artificial chromosome (BAC) reporter lines in zebrafish, we present the expression patterns of teneurin 1-4 across the central nervous system and identify some of the teneurin-positive cell types, focusing particularly on the visual system.

\section{MATERIALS AND METHODS}

\section{Zebrafish Husbandry}

Zebrafish (Danio rerio) adults and embryos were maintained in accordance with the Animals (Scientific Procedures) Act 1986 under license from the United Kingdom Home Office (PPL70/9036).

Pairwise zebrafish spawnings were set up using Ekkwill wild type adults (Ekkwill Breeders, Florida). Larvae were maintained between 0 and 5 days post fertilization $(\mathrm{dpf})$ at $28.5^{\circ} \mathrm{C}$ on a $14 \mathrm{~h}$ $\mathrm{ON} / 10 \mathrm{~h}$ OFF light cycle in $1 \times$ Danieau solution $(58 \mathrm{mM} \mathrm{NaCl}$, $0.7 \mathrm{mM} \mathrm{KCl}, 0.6 \mathrm{mM} \mathrm{Ca}\left(\mathrm{NO}_{3}\right)_{2}, 0.4 \mathrm{mM} \mathrm{MgSO}_{4}, 5 \mathrm{mM}$ HEPES) supplemented with PTU (Sigma) at a final concentration of $200 \mu \mathrm{M}$. Where necessary, chorions were removed using forceps. Larvae were anesthetized with MS222 and fixed overnight with $4 \%$ paraformaldehyde (PFA) in $\mathrm{PBS}$ at $4^{\circ} \mathrm{C}$, then dehydrated in $100 \%$ methanol and stored at $-20^{\circ} \mathrm{C}$. 


\section{RNA Probe Synthesis}

cDNAs spanning several exons of each tenm were blunt cloned into the pSC-B-Amp plasmid using the following primers: tenm1 exons 19-22 Fwd: gatctcagcaggaatgtggagg, Rev: cagcatccogcgttactgatg; tenm2 exons 27-29 Fwd: gcatgtgttcaaccactcca, Rev: gcgccatttaacaccagaac; tenm3 exons 2628 Fwd: gggactatgacattcaagcaggtc, Rev: cattgttggcactgtcggccag; tenm4 exons 21-25 Fwd: catttcccagcagcctccagtc, Rev: ctttcgcgtagccgtcgtcg. Plasmids were linearized and transcribed using NotI and T3 (for tenm1, tenm3, tenm4) or EcoRV and T7 (for tenm2) to generate DIG-labeled anti-sense riboprobes. Probes were purified by $\mathrm{LiCl}$ precipitation and suspended in ultrapure water. Working probes were diluted to $250 \mathrm{ng} / \mathrm{ml}$ in hybridization mix $(\mathrm{HM})$ containing $50 \%$ formamide, $5 \times$ SSC $\mathrm{pH}$ 6.0, 0.1\% Tween-20, $50 \mu \mathrm{g} / \mathrm{ml}$ Heparin and $500 \mu \mathrm{g} / \mathrm{ml} \mathrm{tRNA}$.

\section{Wholemount in situ Hybridization}

In situ hybridization was performed according to Thisse and Thisse (2008). In brief, samples were rehydrated through a series of 75,50 , and $25 \%$ methanol, into PBS containing $0.1 \%$ Tween-20 (PBTw) and permeabilized by digestion with $10 \mu \mathrm{g} / \mathrm{ml}$ Proteinase $\mathrm{K}$ in PBTw at room temperature. Digestion times varied according to developmental stage $(10 \mathrm{~min}$ for $1 \mathrm{dpf}, 20 \mathrm{~min}$ for $2 \mathrm{dpf}$ and $30 \mathrm{~min}$ for 3-5 dpf). Digestion was stopped by post-fixing the embryos in 4\% PFA in PBS for $20 \mathrm{~min}$ at room temperature. Residual fix was removed by washing $2 \times 10 \mathrm{~min}$ in PBTw before embryos were prehybridized in $\mathrm{HM}$ at $70^{\circ} \mathrm{C}$ for $3 \mathrm{~h}$. Probes were hybridized overnight at $70^{\circ} \mathrm{C}$ and the embryos were subsequently washed $1 \times 10 \mathrm{~min}$ through 75,50 , and $25 \% \mathrm{HM}$, into $2 \times$ SSC followed by $2 \times 30 \mathrm{~min}$ washes with $0.2 \times \mathrm{SSC}$, all at $70^{\circ} \mathrm{C}$. Then, at room temperature, the larvae were washed through a series of 75,50 , and $25 \% 0.2 \times$ SSC, into PBTw before non-specific binding was blocked for $3 \mathrm{~h}$ at room temperature using $2 \mathrm{mg} / \mathrm{ml} \mathrm{BSA}$ and $2 \%$ sheep serum in PBTw. Samples were incubated overnight at $4^{\circ} \mathrm{C}$ in blocking buffer containing anti-DIG antibody (Roche) diluted 1:10,000. Excess antibody was removed by washing $4 \times 1 \mathrm{~h}$ in PBTw. Finally, samples were washed $2 \times 15 \mathrm{~min}$ in staining buffer containing $10 \mathrm{mM}$ Tris- $\mathrm{HCl} \mathrm{pH} 9.5,100 \mathrm{mM} \mathrm{NaCl}$ and $0.1 \%$ Tween-20 and color staining was developed in the dark at room temperature using NBT/BCIP stock solution (Roche) diluted in staining buffer. The color reaction was checked frequently and stopped by washing in PBTw. Images were captured using a Leica M165 FC stereomicroscope, QImaging Retiga camera and Volocity software.

\section{Paraffin Sections}

Larvae were post-fixed overnight in 4\% PFA at room temperature and dehydrated for $30 \mathrm{~min}$ in $100 \%$ methanol and $30 \mathrm{~min}$ in isopropanol. Samples were cleared with tetrahydronaphthalene (THN) for $30 \mathrm{~min}$. Molten Paraplast wax (Sigma) was added to give a $1: 1$ mix of THN:wax and incubated at $60^{\circ} \mathrm{C}$ for $30 \mathrm{~min}$. This was replaced $3 \times 1 \mathrm{~h}$ with fresh molten wax at $60^{\circ} \mathrm{C}$ before samples were oriented, embedded and left to solidify. $12 \mu \mathrm{m}$ transverse sections were cut on a Microm HM315 microtome and mounted on slides treated with glycerin albumin. Slides were dried overnight at $37^{\circ} \mathrm{C}$ and dewaxed by $2 \times 4 \mathrm{~h}$ washes in Histoclear II. Coverslips were mounted using a 1:3 mixture of Histoclear II: Canada Balsam. Images were captured using an Olympus Vanox-T with 40 objectives and a QImaging Retiga 2000R camera with QCapture Pro software.

\section{BAC Transgenesis}

The BAC clones containing the gene sequences for tenm 1, 2.14 and 4 were identified by using the ENSEMBL database of the Wellcome Trust Sanger Institute (Daniokey library reference: tenm1, DKEY-275B22; tenm2.14, DKEY-47H20; tenm4, DKEY84I3). Zebrafish have two genes that encode for tenm2, tenm2.14 and tenm2.21, present on chromosomes 14 and 21, respectively (Supplementary Figure S1). The tenm2.14 paralog was chosen for further investigation, based on its higher resemblance to other tenm 2 orthologs. BAC clones were transformed first with a pRed-Flp4 plasmid before a recombineering step was used to insert a membrane-localized reporter gene (mCitrine) together with a kanamycin resistance cassette at the beginning of the tenm gene. Transient transgenic zebrafish lines were created by microinjection of teneurin:mCitrine BAC constructs into onecell stage embryos, before embryos were allowed to develop at $28.5^{\circ} \mathrm{C}$ with PTU until 3-5 dpf. Embryos were mounted laterally on glass slides using $1 \%$ low-melting point agarose in Danieau's solution and imaged on an LSM 710 Zeiss confocal equipped with a spectral detection scan head and a $20 \times / 1.0$ NA waterimmersion objective.

\section{RESULTS}

\section{Tenm1-4 Are Widely Expressed Across the Central Nervous System}

The expression patterns of the four vertebrate paralogs of teneurin were investigated via wholemount in situ hybridization on zebrafish embryos over 1 to $5 \mathrm{dpf}$. Tenm 1 is first detected in a discrete spot in the ventral midbrain at $1 \mathrm{dpf}$ (Figure 2A; arrow). Later expression is more widespread but localized anteriorly with tenm 1 observed in multiple regions of the forebrain, midbrain and hindbrain at 2-5 dpf (Figure 2). Similarly, significant tenm 2 expression is first detected anteriorly at $2 \mathrm{dpf}$ in the olfactory bulbs and in multiple clusters of neurons in the midbrain and hindbrain, persisting over 3-5 dpf (Figure 3).

In contrast to tenm1 and 2, which were either weak or absent at $1 \mathrm{dpf}$, strong tenm 3 expression is already detected early at $1 \mathrm{dpf}$ in the forebrain and midbrain, as well as the developing retina (Figures $\mathbf{4 A}, \mathbf{F}, \mathbf{K}$ ). By $2 \mathrm{dpf}$, high expression is specifically localized to the optic tectum, ventral retina, medulla oblongata and tips of the fin buds, persisting over 3-5 dpf (Figure 4). Similarly, strong tenm 4 expression is also detected early at $1 \mathrm{dpf}$ in the forming retina, midbrain and hindbrain, and becomes localized to the inner layers of the retina, olfactory bulbs, optic tectum, subset of hindbrain neurons, and along the midhindbrain boundary at 2-3 dpf. This expression persists over 4-5 dpf (Figure 5). 


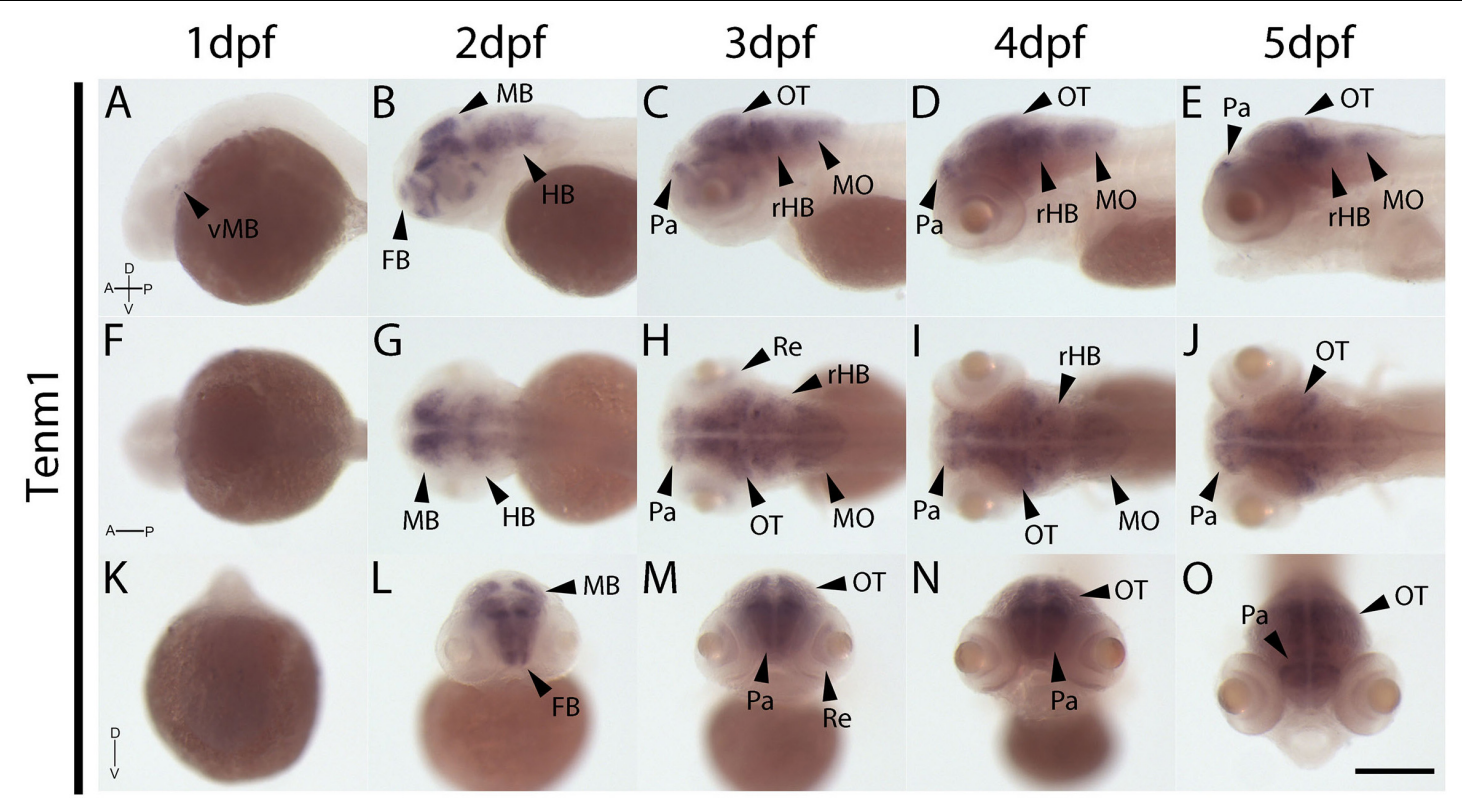

FIGURE 2 | Wholemount expression of tenm1 during zebrafish development. Tenm1 expression during 1-5 dpf shown from lateral (A-E), dorsal (F-J) and frontal (K-O) perspectives. A, Anterior; D, Dorsal; FB, Forebrain; HB, Hindbrain; MB, Midbrain; MO, Medulla Oblongata; OT, Optic Tectum; Pa, Pallium; P, Posterior; Re, Retina; rHB, rostral Hindbrain; V, Ventral; vMB, ventral Midbrain. Scale bar in all panels $=250 \mu \mathrm{m}$.

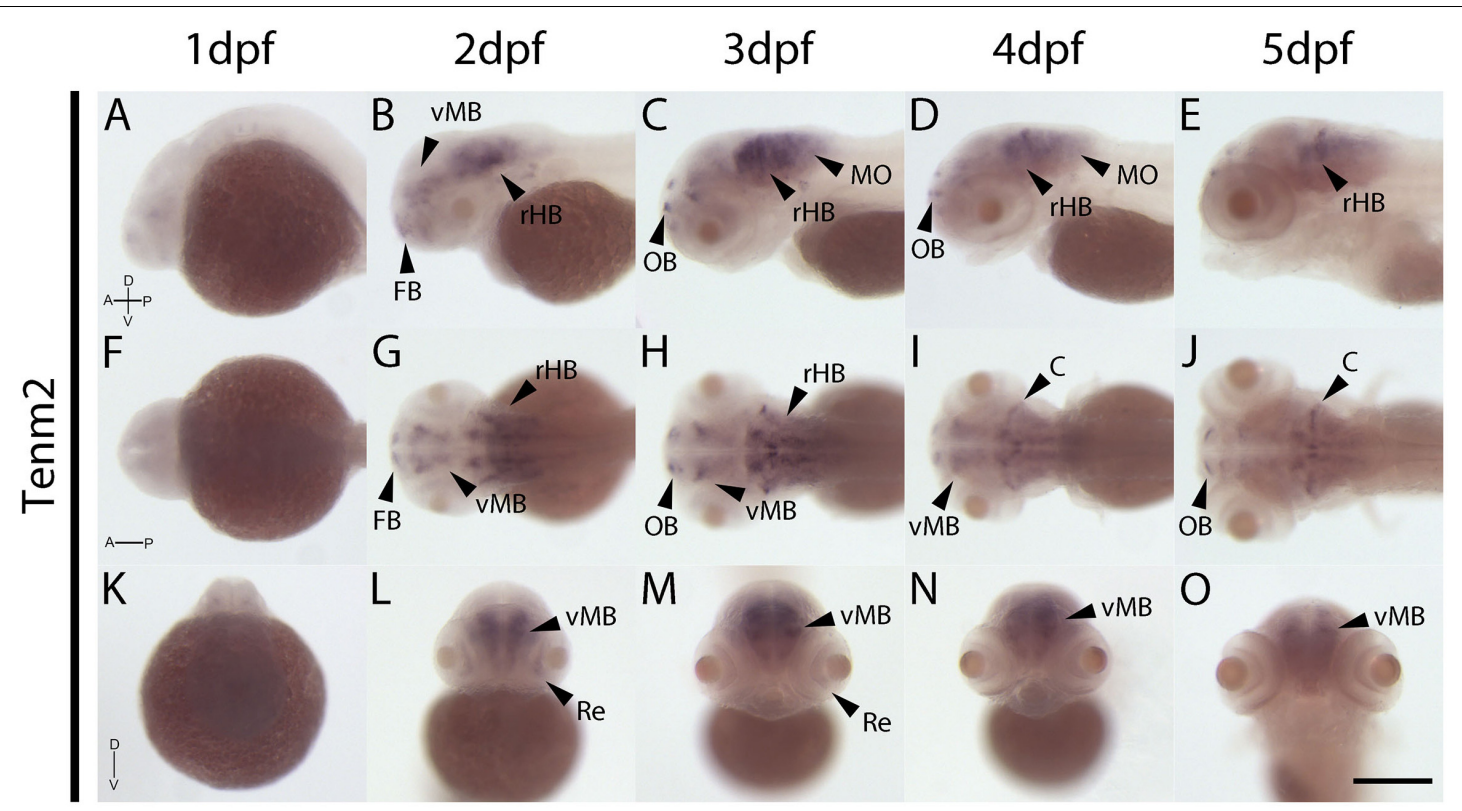

FIGURE 3 | Wholemount expression of tenm2 during zebrafish development. Tenm2 expression during 1-5 dpf shown from lateral (A-E), dorsal (F-J) and frontal (K-O) perspectives. A, Anterior; C, Cerebellum; D, Dorsal; FB, Forebrain; MO, Medulla Oblongata; OB, Olfactory Bulb; P, Posterior; Re, Retina; rHB, rostral Hindbrain; V, Ventral; vMB, ventral Midbrain. Scale bar in all panels $=250 \mu \mathrm{m}$.

\section{The Teneurins Are Expressed Across Interconnected Regions of the Zebrafish Nervous System During Development}

Transverse sections across the zebrafish central nervous system were collected from wholemount in situ hybridization samples in order to gain more detailed insight into the expression pattern of teneurins from 1 to $4 \mathrm{dpf}$.

At $1 \mathrm{dpf}$, tenm 1 is only expressed in a discrete population of cells in the ventral midbrain (Figure 6AB; arrow), while tenm2 expression is absent. In contrast, tenm 3 and tenm 4 are expressed strongly in broad domains of the forming retina and midbrain 
(Figures 6CA, DA, CB, DB). Tenm4 is expressed throughout the rostral and caudal hindbrain (Figures 6DC, DD), whereas tenm3 is restricted laterally (Figures 6CC, CD). While tenm 4 is strongly expressed in the neural tube (Figure 6DE), tenm 3 expression is much weaker (Figure 6CE).
By $2 \mathrm{dpf}$, tenm 3 and tenm 4 are expressed strongly in the forebrain pallium (Figures 7CA, DA), while tenm 1 transcripts are only faintly detected (Figure 7AA). In the retina, tenm3 is restricted to retinal progenitors in the inner ventral domain (Figure 7CB), while tenm 4 is expressed more broadly across

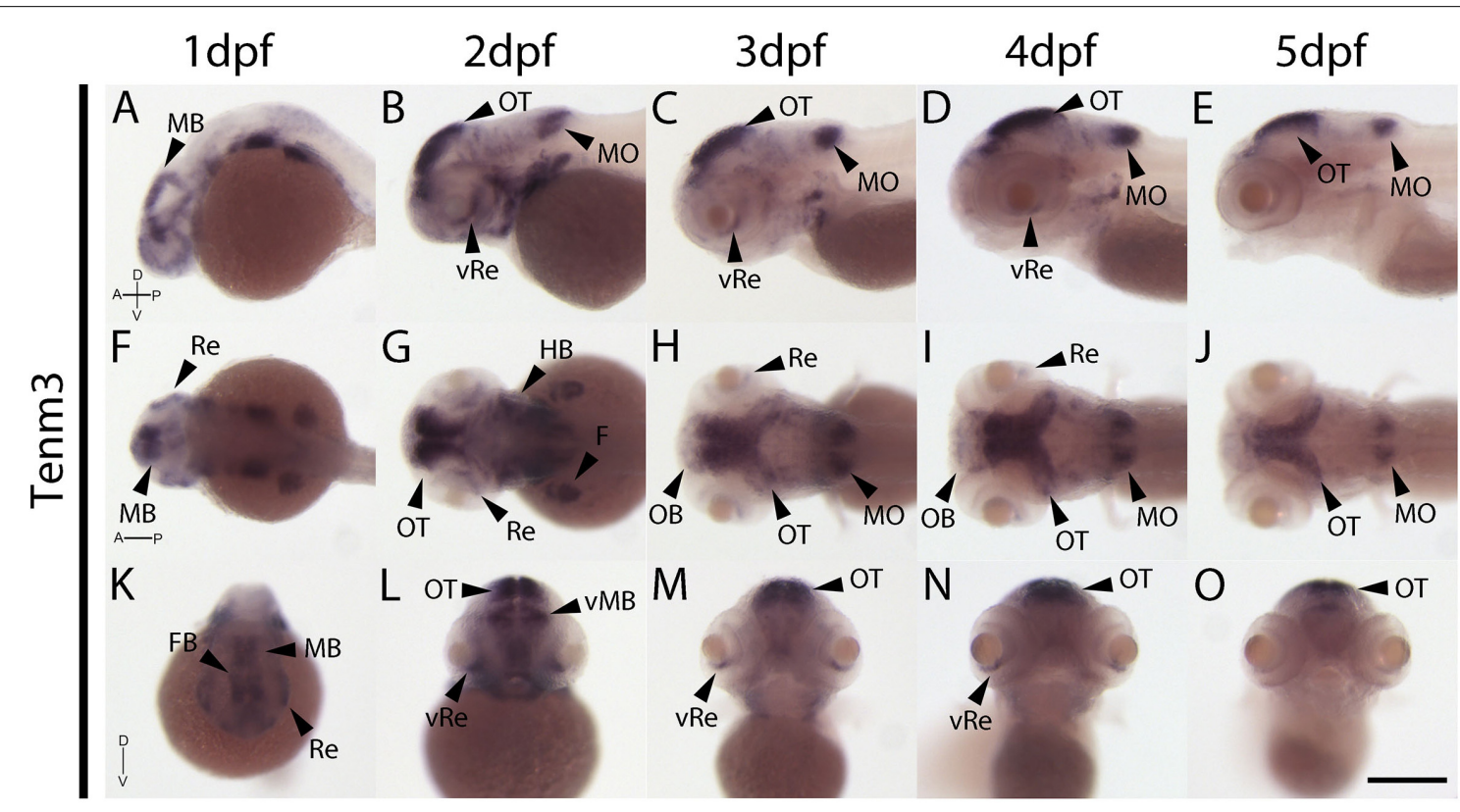

FIGURE 4 | Wholemount expression of tenm3 during zebrafish development. Tenm3 expression during 1-5 dpf from lateral (A-E), dorsal (F-J) and frontal (K-O) perspectives. A, Anterior; D, Dorsal; F, Fin; FB, Forebrain; HB, Hindbrain; MB, Midbrain; MO, Medulla Oblongata; OB, Olfactory Bulb; OT, Optic Tectum; P, Posterior; Re, Retina; V, Ventral; vRe, ventral Retina. Scale bar in all panels $=250 \mu \mathrm{m}$.

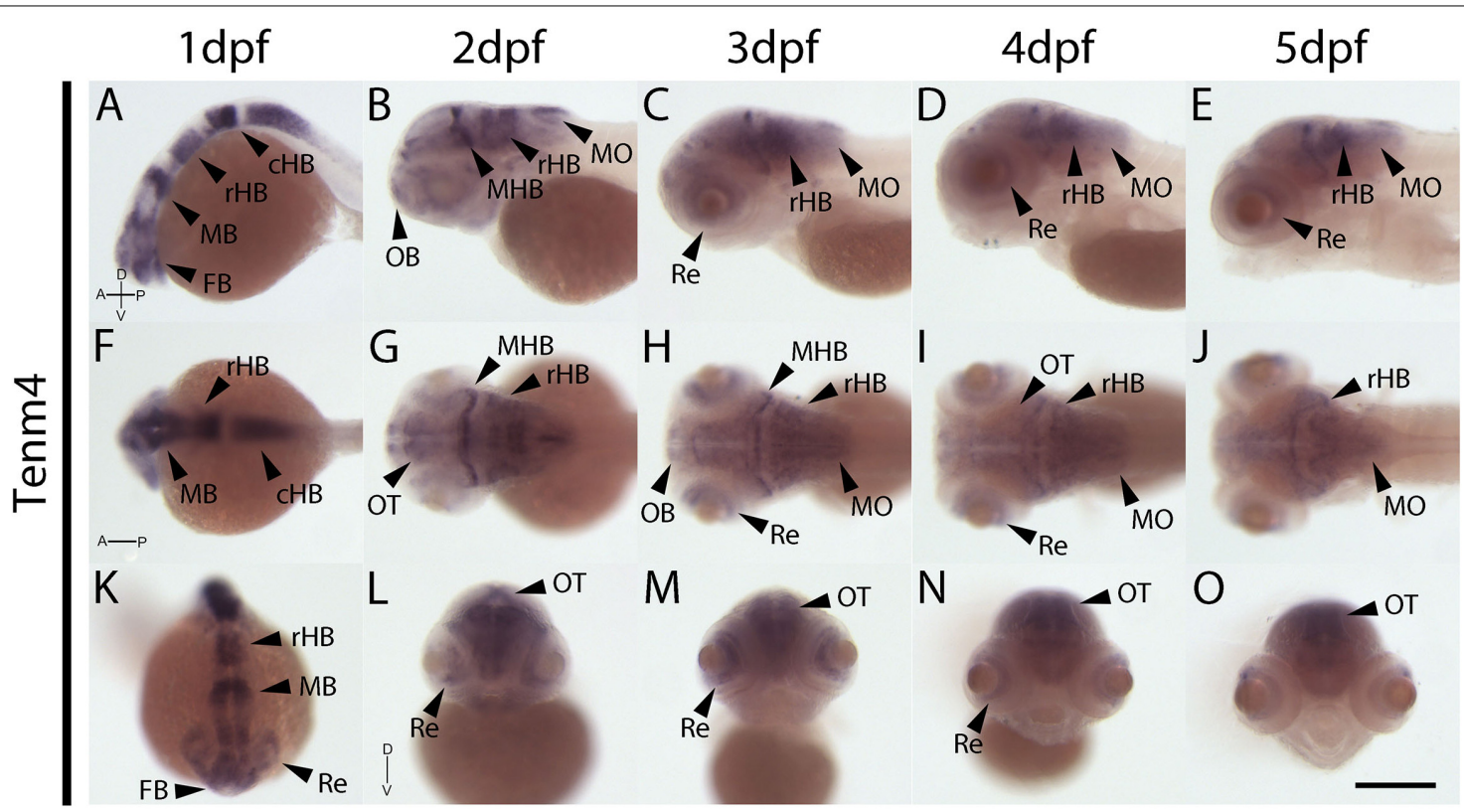

FIGURE 5 | Wholemount expression of tenm4 during zebrafish development. Tenm4 expression during 1-5 dpf from lateral (A-E), dorsal (F-J) and frontal (K-O) perspectives. A, Anterior; cHB, caudal Hindbrain; D, Dorsal; FB, Forebrain; MB, Midbrain; MHB, Mid-Hindbrain Boundary; MO, Medulla Oblongata; OB, Olfactory Bulb; OT, Optic Tectum; P, Posterior; rHB, rostral Hindbrain; Re, Retina; V, Ventral. Scale bar in all panels $=250 \mu \mathrm{m}$. 
the retina and at the ciliary marginal zone (Figure 7DB). Neither tenm1 nor tenm2 are detected in the retina at these stages (Figures $7 \mathbf{A B}, \mathbf{B B}$ ). Tectal cells express tenm4 broadly (Figure 7DC), whereas tenm 3 is restricted dorsally (Figure 7CC). A weak expression of tenm 1 can also be observed in the lateral tectum (Figure 7AC), and overlapping tenm2, 3 and 4 expression (Figures 7BD, CD, DD) is detected in the cerebellum of the rostral hindbrain, but only tenm 3 and tenm 4 are observed at the rhombic lip and medulla oblongata of the caudal hindbrain (Figures 7CE, DE). No teneurin expression is detected in the spinal cord at $2 \mathrm{dpf}$.

Tenm 1 and tenm 2 are not detected in the forebrain, retina or midbrain at $3 \mathrm{dpf}$. Tenm 3 and tenm 4 can be detected in the pallium and subpallium, but the latter only weakly (Figures 8CA, DA). Tenm3 and tenm4 are also detected in amacrine cells, retinal ganglion cells (Figures 8CB, DB) and in tectal neurons (Figures 8CC, DC). All teneurins are expressed in the rostral and caudal hindbrain (Figures 8AD, AE, BD, BE, CD, CE, DD, DE) but in different layers of the cerebellum and medulla oblongata, with tenm 1 and tenm 2 expression weak in this area. Tenm 3 and tenm 4 are also present at the rhombic lip, while again, no teneurin expression is observed along the spinal cord.
Finally, at $4 \mathrm{dpf}$, tenm 3 and tenm 4 expression persists in the pallium (Figures 9CA, DA), while in the retina, tenm3 mRNA is no longer detected in amacrine cells and remains only in the ventral population of RGCs (Figure 9CB). Tenm4 expression in these cells is also reduced compared to $3 \mathrm{dpf}$ (Figure 9DB), while both tenm 3 and tenm 4 are present in tectal cells (Figures 9CC, DC), as well as in the cerebellum, rhombic lip and medulla oblongata (Figures 9CD, CE, DD, DE). Very weak tenm 1 expression overlaps in the cerebellum (Figure 9AD). Tenm 2 expression is too weak to detect in sections at $4 \mathrm{dpf}$.

Although teneurin 1-4 were found to be widely expressed in overlapping regions of the nervous system, we cannot infer from our studies whether different teneurin paralogs are co-expressed within individual cells.

\section{The Teneurins Are Expressed in Multiple Cell Types of the Retina}

To further investigate the types of retinal cells that are positive for individual teneurins, we generated BAC constructs for tenm1, 2.14 and 4, inserting the coding sequence for the fluorescent reporter mCitrine at the place of the ATG in the genomic locus (Supplementary Figure S1).

We have previously described the expression of tenm3 in the zebrafish visual system in detail, including the identification

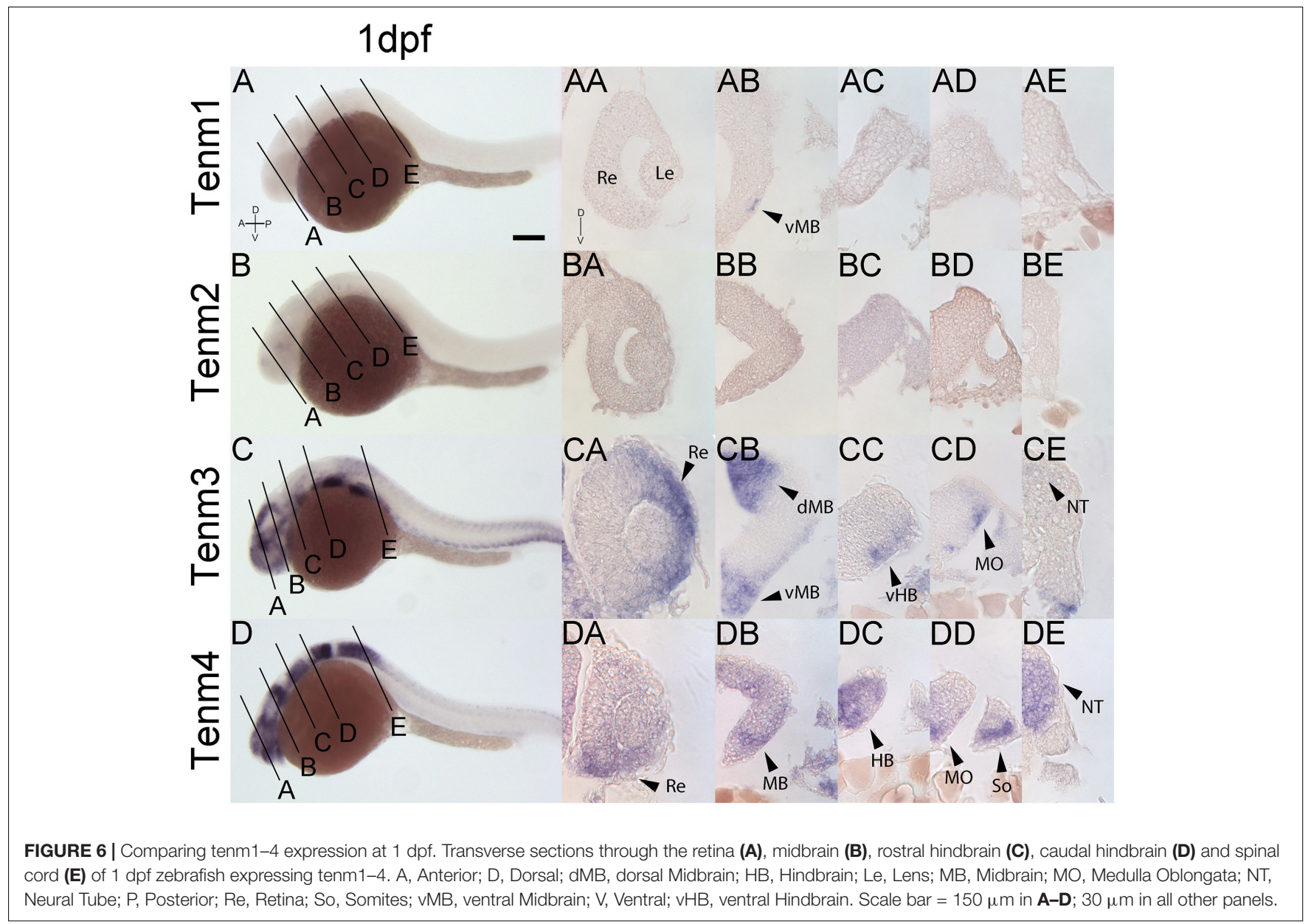


of tenm3-positive retinal cells using a BAC transgenic strategy (Antinucci et al., 2013, 2016). Here we extend these analyses to describe retinal and tectal cells in zebrafish positive for other members of the teneurin family. Transient transgenic zebrafish embryos expressing teneurin:mCitrine BAC constructs were imaged at 3-5 $\mathrm{dpf}$ to investigate the morphology and distribution of tenm1-, tenm2.14- and tenm4-positive cells in the developing visual system, where teneurins have been shown to play a key role in establishing functional neural circuitry. All three BAC constructs tested labeled single cells of the visual system, including RGCs, amacrine cells and tectal cells (Figure 10A).

The tenm1:mCitrine BAC construct consistently labeled RGCs (Figures 10B-F) and a low number of amacrine cells in the retina, as well as cells in the optic tectum. All tenm1-positive tectal cells shared a characteristic elongated morphology with the axon projecting from the dendritic arbor instead of from the cell soma (Figures 10G,H). Citrine-expressing RGCs in the retina were classified morphologically into three main groups according to their arborization patterns (Figure 10I); monostratified, bistratified or diffuse. Monostratified RGCs were further subdivided into $\mathrm{ON}$, OFF and asymmetric dendritic arbors according to the distance of the dendritic field to the cell body. Although all RGC types had similar dendritic field diameters, the monostratified asymmetric and diffuse asymmetric RGCs had the largest dendritic field coverage/area (Table 1).

In direct contrast to tenm 1:mCitrine, the tenm2.14:mCitrine BAC construct labeled predominantly amacrine cells (Figures $11 \mathrm{~A}-\mathrm{J}$ ) along with a very low number of RGCs. Tectal cells were also labeled (Figure $\mathbf{1 1 K}$ ), and similar to when the tenm1:mCitrine construct was used, the number of labeled cells was higher in the optic tectum than in the retina. For Tenm2.14:mCitrine, the fluorescently labeled cells could be classified into narrow-field, medium-field and wide-field amacrine cells according to their dendritic arborization and morphology, with further subdivision in accordance to their stratification within the inner plexiform layer (IPL); ON or OFF (Figure 11L). A total number of 9 amacrine cell types were defined: 6 narrow-field ( 2 monostratified and 4 diffuse), 2 medium-field (1 monostratified and 1 diffuse) and one wide-field (monostratified), with the narrow-field amacrine cells being the most abundant. The average dendritic field for narrow-field amacrine cells was 4 times and 14 times smaller than the average

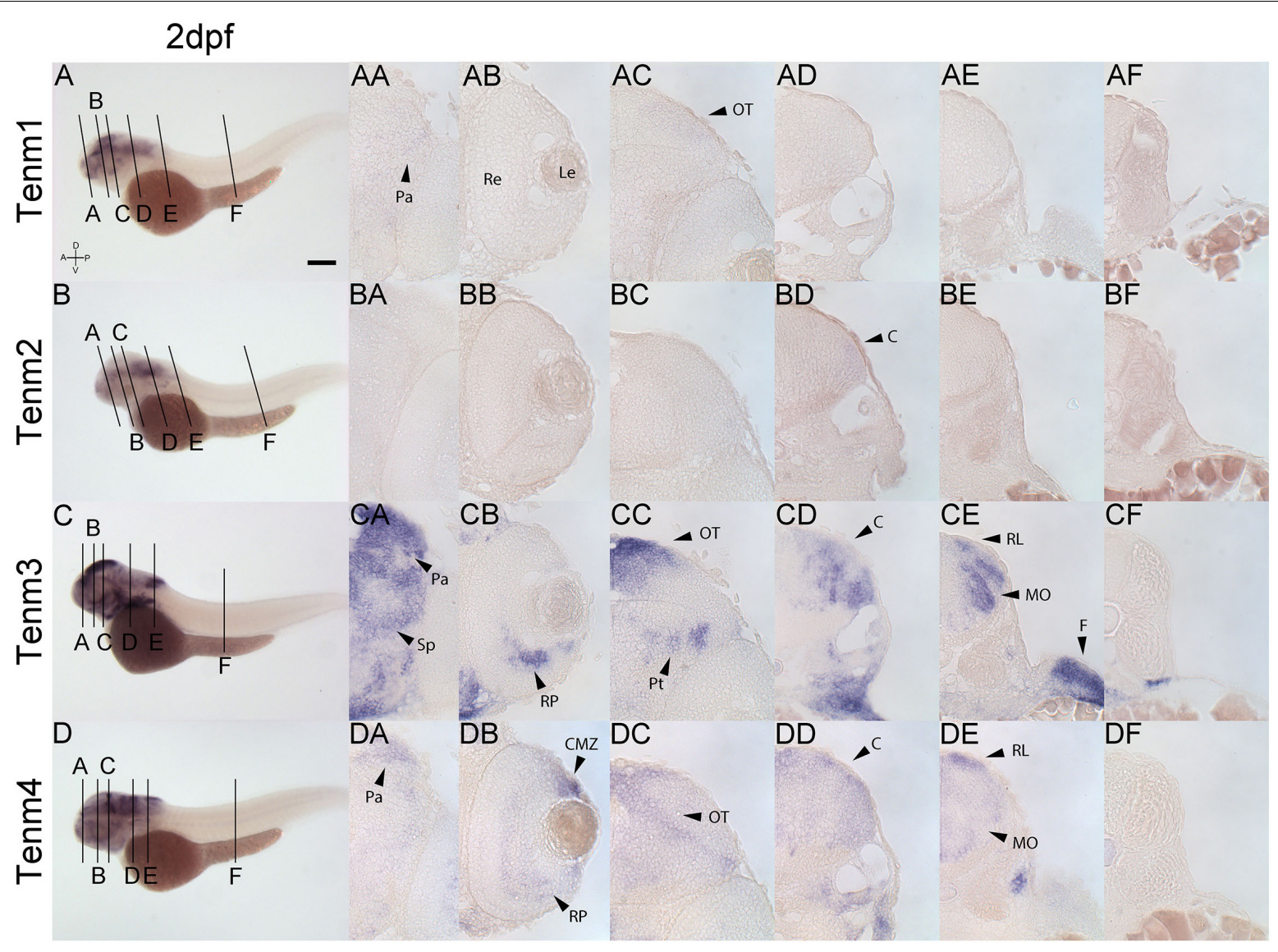

FIGURE 7 | Comparing tenm1-4 expression at 2 dpf. Transverse sections through the forebrain (A), retina (B), midbrain (C), rostral hindbrain (D), caudal hindbrain (E) and spinal cord (F) of 2 dpf zebrafish expressing tenm1-4. A, Anterior; C, Cerebellum; CMZ, Ciliary Marginal Zone; D, Dorsal; F, Fin; Le, lens; MO, Medulla Oblongata; OT, Optic Tectum; Pa, Pallium; P, Posterior; Pt, Pretectum; Re, Retina; RL, Rhombic Lip; Rp, Retinal Progenitors; Sp, Subpallium; V, Ventral. Scale bar $=250 \mu \mathrm{m}$ in A-D; $30 \mu \mathrm{m}$ in all other panels. 


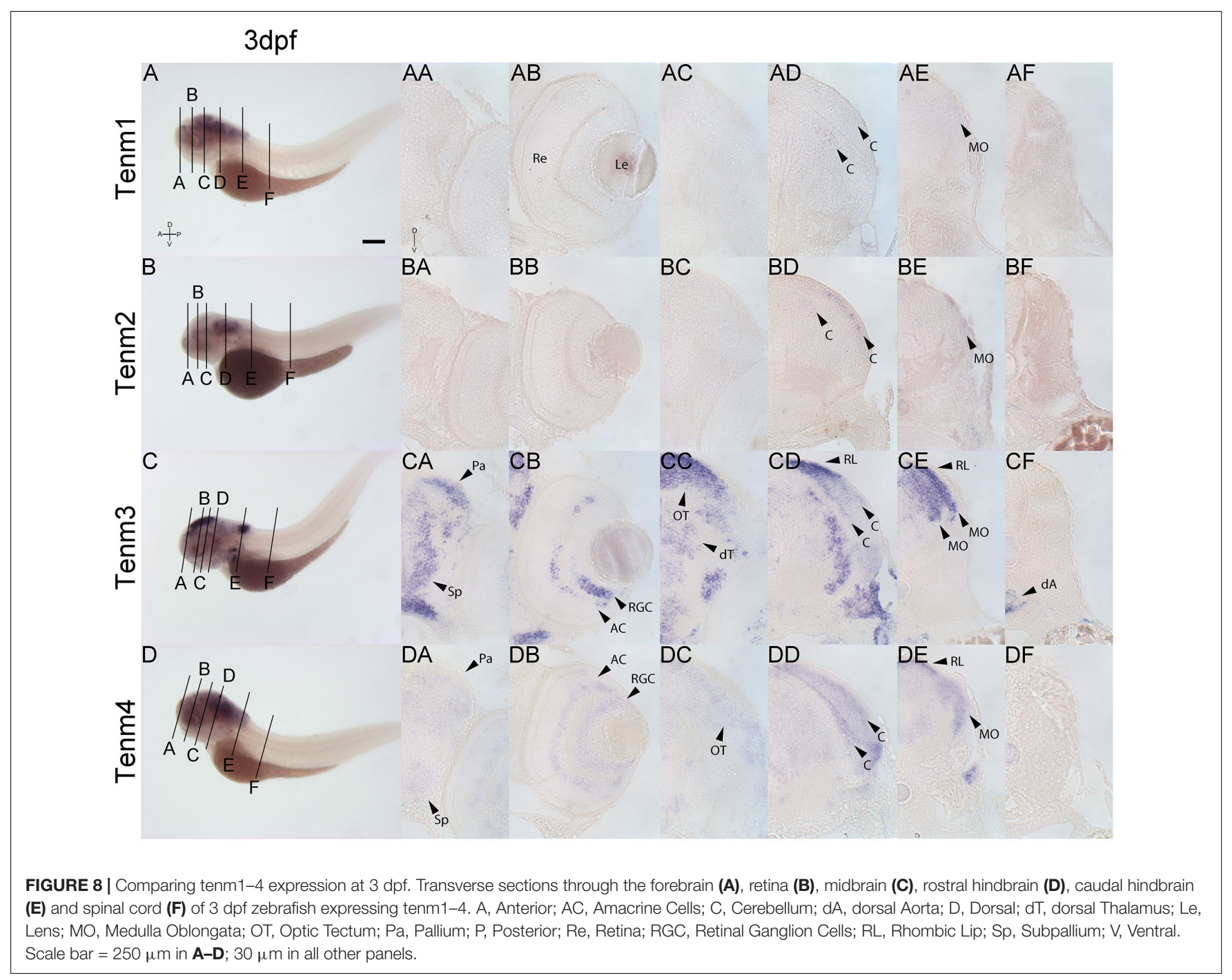

dendritic field of medium-field amacrine cells and wide-field amacrine cells, respectively (Table 2).

While the tenm1:mCitrine and tenm2.14:mCitrine BAC constructs strongly labeled a range of distinct cell types, the tenm4:mCitrine BAC only lead to weak reporter expression in cells and further detailed morphological analyses could not be carried out.

\section{DISCUSSION}

We describe here the expression pattern of different members of the teneurin family across the central nervous system in zebrafish during development, focusing particularly on the visual system where they have been shown to play a pivotal role in establishing connectivity, via in situ hybridization and BAC transgenesis.

Although the teneurins are also expressed in non-neuronal tissues, such as at sites of cell migration and at muscle attachment points, they are primarily concentrated in the central nervous system and at sites of pattern formation during development
(Baumgartner and Chiquet-Ehrismann, 1993; Baumgartner et al., 1994; Fascetti and Baumgartner, 2002; Drabikowski et al., 2005). This localization is observed across all teneurin expressing species and supports the role of teneurins in neurodevelopmental processes such as neurite outgrowth, axon guidance and synapse formation (Tucker and Chiquet-Ehrismann, 2006; Leamey and Sawatari, 2014). Consistent with that, we observed an early widespread expression of teneurins across the central nervous system with tenm 1,3 and 4 expressions already present in embryos at $1 \mathrm{dpf}$. Indeed, the early strong expression of tenm 3 and 4 in the developing retina supports the role of teneurins, particularly of tenm3, in directing the functional connectivity of RGCs and amacrine cells in the developing visual system (Antinucci et al., 2013). Both genes remain strongly expressed in various structures of the developing central nervous system well into $4 \mathrm{dpf}$. Tenm 1 and 2 expression, on the other hand, is not observed at all in the retina during development and overall expression is weak, especially by $4 \mathrm{dpf}$.

All four teneurins are also expressed at various levels in different layers of the cerebellum at $3 \mathrm{dpf}$. Interestingly, 


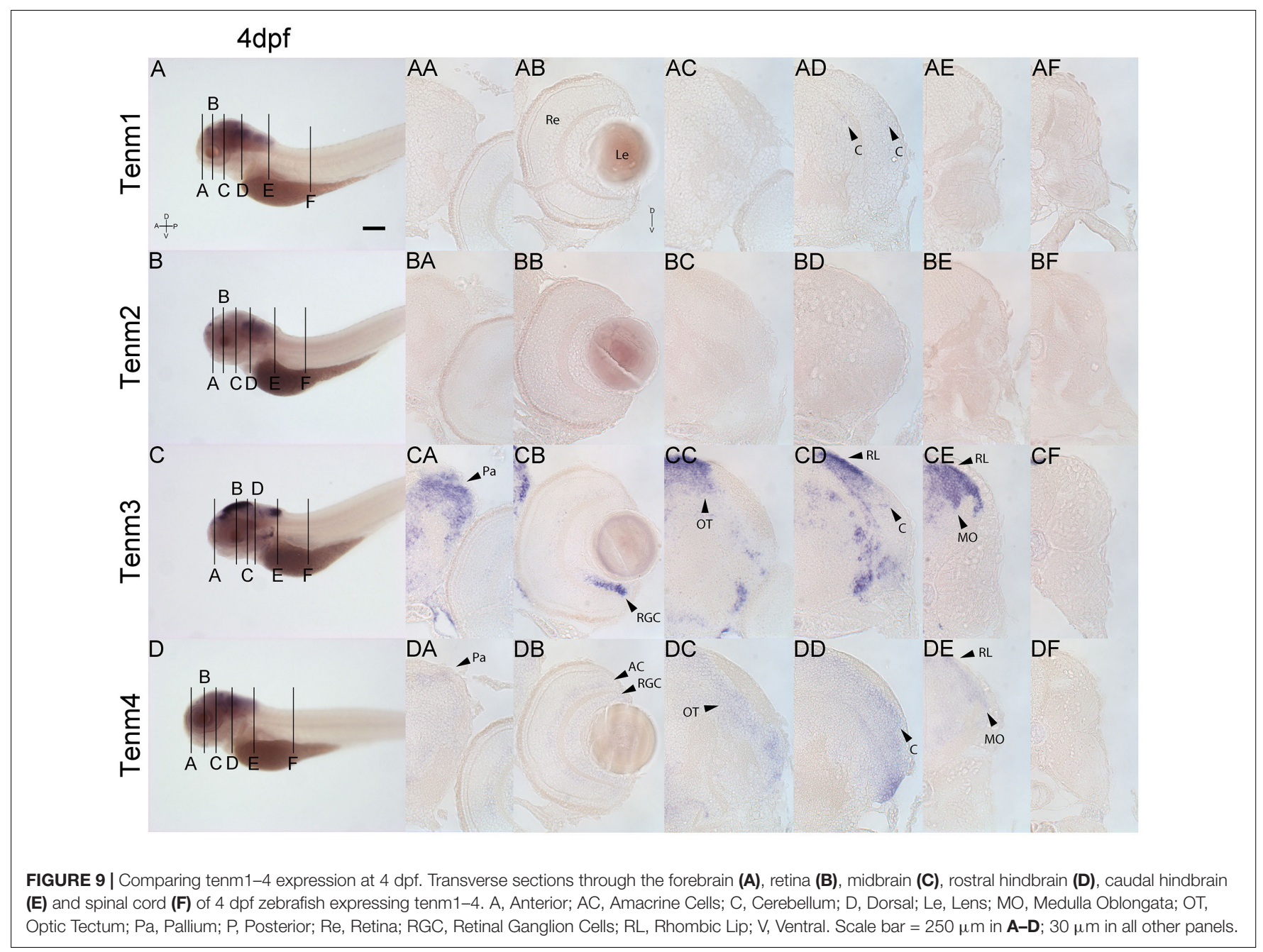

mutations in tenm 3 have been implicated as a plausible candidate for a new dominantly inherited form of pure adult-onset cerebellar ataxia in humans (Storey et al., 2009). Essentially, all four teneurin paralogs are expressed in an overlapping manner across interconnected areas of the central nervous system of the zebrafish embryo, such as the retina and the optic tectum. The strong co-expression of tenm 3 and 4 across many areas may be suggestive of a possible functional redundancy between the two teneurins, although this is still to be further investigated. Mieda et al. (1999), also performed in situ hybridization studies on tenm 3 and 4 in the developing zebrafish embryo and showed that the expression patterns were complementary to each other early on in the developing embryo $(<26 \mathrm{hpf})$ but becomes ambiguous at later stages.

While we are one of the first to present an in-depth study of the expression pattern of all four teneurin paralogs across the zebrafish embryo during development, comprehensive expression data is already available for other species such as the mouse. For example, Zhou et al. (2003) showed that the expression of the four teneurin genes in the developing and adult central nervous system of mice was distinct but partially overlapping. Similar to what we found in the zebrafish, the teneurins were expressed widely across the central nervous system and significant levels of tenm 3 and 4 were detected early on during development (Zhou et al., 2003). All four teneurins show a graded expression across the cortex and striatum during embryonic and early postnatal stages (Li et al., 2006; BibolletBahena et al., 2017), and intriguingly, tenm4 is also expressed in the subventricular zone, suggesting a possible role in determining cortical progenitor cell fate (Li et al., 2006).

While tenm 3 is expressed in interconnected areas of the zebrafish visual system such as the retina and optic tectum, similarly, in the mouse visual system, tenm 3 is expressed across interconnected regions of the retina, dLGN, superior colliculus (SC) and visual cortex (Leamey et al., 2007, 2008). Tenm2 and tenm 4 in mice have also been shown to be expressed strongly in the interconnected visual cortex and dLGN (Li et al., 2006). Mouse hippocampal tenm expression also matches the topographic connectivity between the entorhinal cortex, CA1 and subiculum (Berns et al., 2018).

Zhou et al. (2003) also noted a differential distribution of mRNA transcripts and protein, which has also been previously observed in Drosophila, and which they explain as possible axonal transport into target regions (Baumgartner et al., 1994; Zhou 

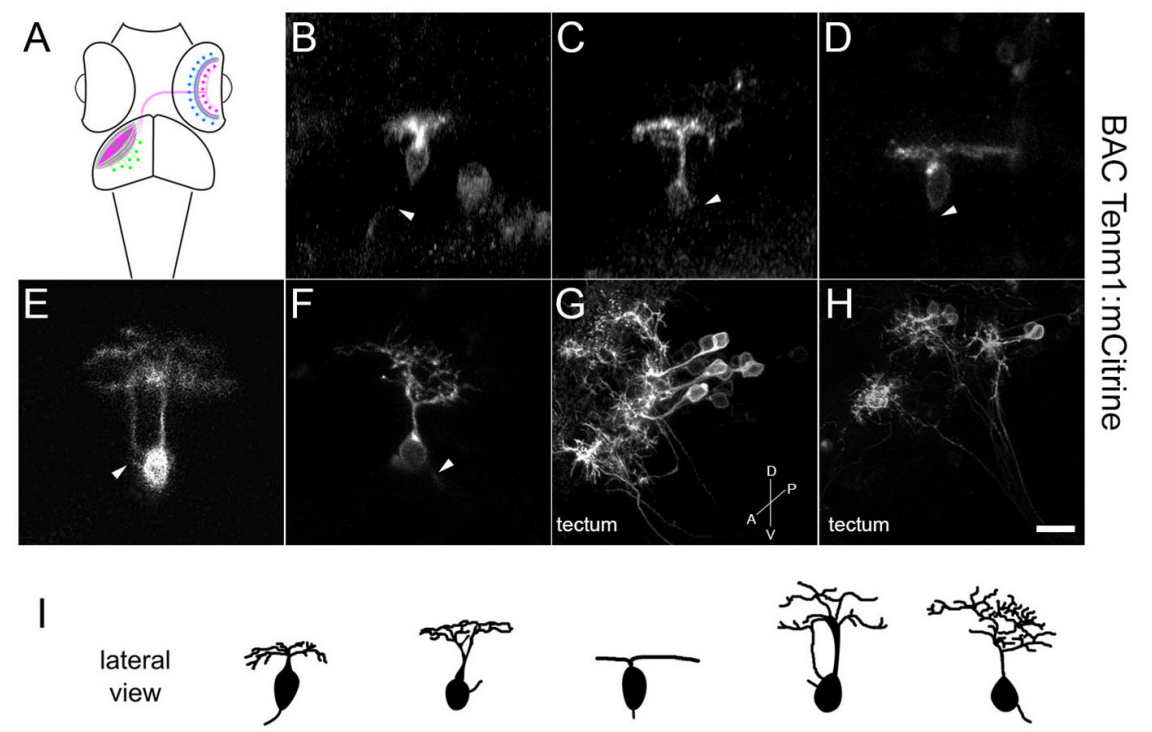

view
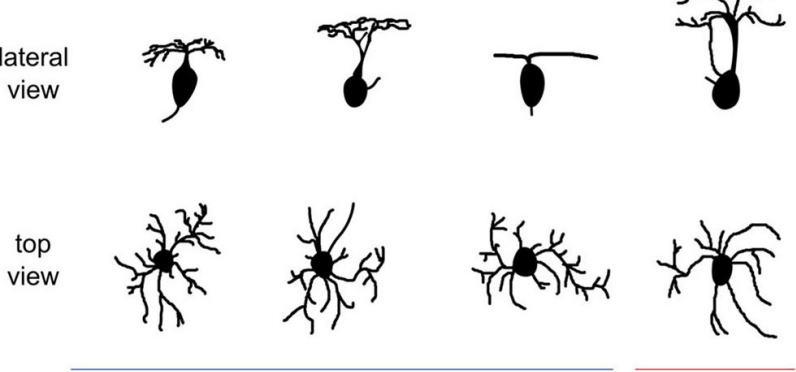

bistratified

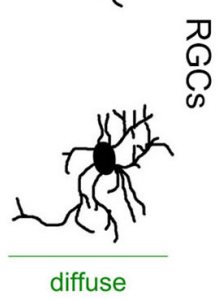

FIGURE 10 | Tenm1-positive cells in the zebrafish visual system in tenm1:mCitrine transient transgenic fish at 3 dpf. Schematic of amacrine cells (blue), RGCs (magenta) and tectal cells (green) in the zebrafish visual system (A). Lateral view of representative RGCs with monostratified (B-D), bistratified (E) and diffuse (F) dendritic arbors. RGC axons are marked by arrowheads. Tenm1-positive tectal cells (G-H). All images represent maximum intensity projections of $\sim 30$ Mm confocal z-stacks. Scale bar $10 \mu \mathrm{m}$ (B-F), $30 \mu \mathrm{m}$ (G) and $20 \mu \mathrm{m}$ (H). Schematic representation of tenm1-positive RGC morphology based on representative RGCs imaged in tenm1:mCitrine transient transgenic fish at $3 \mathrm{dpf}(\mathbf{I})$.

TABLE 1 | Mean dendritic field diameter and area of mCitrine-labeled retinal ganglion cells in tenm1-BAC-injected fish.

\begin{tabular}{|c|c|c|c|c|c|}
\hline RGC morphology & Monostratified (ON) & Monostratified (OFF) & Monostratified asymmetric (ON) & Bistratified & Diffuse asymmetric \\
\hline Dendritic field diameter ( $\mu \mathrm{m})$ & $29 \pm 2$ & $27 \pm 4$ & 33 & 31 & $38 \pm 5$ \\
\hline Dendritic field area $\left(\mu \mathrm{m}^{2}\right)$ & $580 \pm 20$ & $600 \pm 200$ & 780 & 720 & $800 \pm 200$ \\
\hline$n$ & 2 & 3 & 1 & 1 & 2 \\
\hline
\end{tabular}

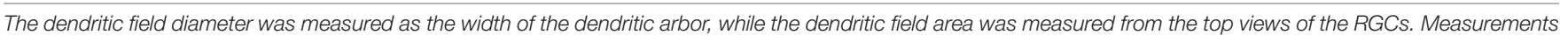
were made with ImageJ. $n$ represents the number of cells analyzed per type. SD is shown for all cell types where $n>1$.

et al., 2003). It would be insightful to investigate this further once reliable antibodies for detecting teneurin protein expression in zebrafish are available.

In the developing chick visual system, tenm 1 and 2 have been found to be predominantly expressed in non-overlapping populations of neurons during the time of axonal growth in embryos (Rubin et al., 1999). Tenm1 is expressed in the IPL, the ganglion cell layer (GCL) and the tectum and displays a complementary pattern of expression with tenm 2 in different sublaminae of the IPL in chick (Kenzelmann et al., 2008). To further investigate teneurin expression in the developing visual system of zebrafish, different teneurin:mCitrine BAC constructs were injected into zebrafish embryos in an attempt to create transient transgenic lines expressing tenm 1, 2.14 and 4. Although tenm 1 and 2 transcript expression was not observed in the retina with in situ hybridization techniques, BAC transgenesis enabled a more sensitive detection of lower levels of teneurin expression.

In addition to validating these BAC constructs, which consistently labeled both amacrine cells and RGCs, a preliminary classification of cells expressing teneurins in the inner retina and optic tectum was accomplished. While the tenm $1: m C i t r i n e$ BAC construct primarily labeled five subtypes of RGCs, the tenm2.14:mCitrine BAC labeled nine amacrine cell subclasses. Tenm4:mCitrine BAC transgenesis was less efficient and exhibited low fluorescence levels so a detailed morphological classification of tenm 4 expressing cells could not be established. Tenm3, on the other hand, has been shown to be expressed by RGCs, amacrine cells and also tectal neurons in zebrafish embryos via in situ hybridization studies (Antinucci et al., 2013), while a BAC transgenic line with Gal4FF under the 

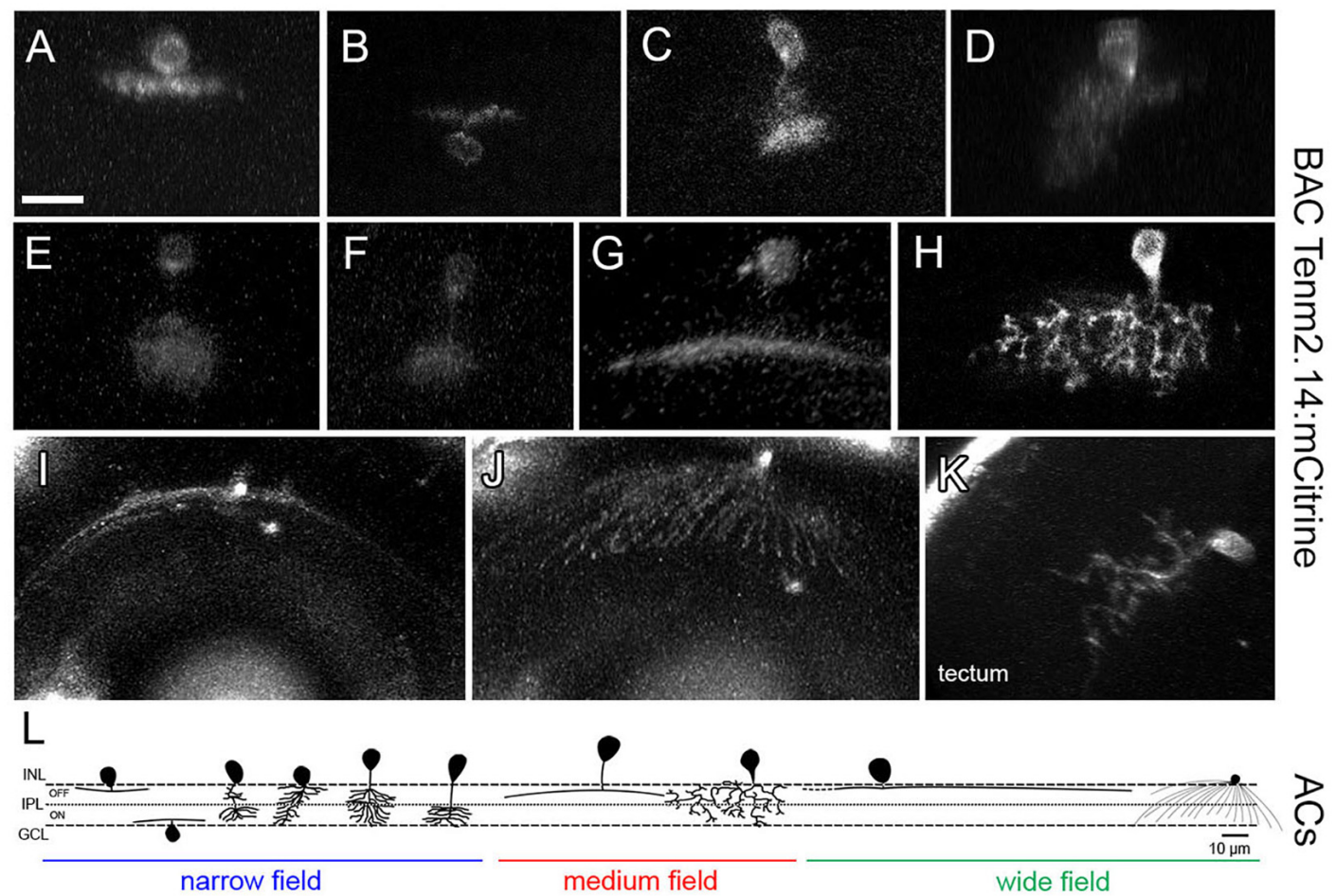

FIGURE 11 | Tenm2.14-positive cells in the zebrafish visual system in tenm2.14:mCitrine transient transgenic fish at 4 and 5 dpf. Lateral view of representative narrow field (A-F), medium field (G-H) and wide field (I) amacrine cells. Top view of a wide field amacrine cell showing ample dendritic coverage of the retina (J). Lateral view of a tectal cell (K). All images represent maximum intensity projections of $\sim 30 \mu \mathrm{m}$ confocal z-stacks. Scale bar $10 \mu \mathrm{m}$ (A-H), $20 \mu \mathrm{m}$ (I-J) and $15 \mu \mathrm{m}$ (K). Schematic representation of tenm2.14-positive amacrine cell morphology based on representative cells imaged in tenm2.14:mCitrine transient transgenic fish at 4 and $5 \mathrm{dpf}$ (L).

transcriptional control of regulatory elements upstream and downstream of the tenm3 start codon, $\operatorname{Tg}$ (tenm3:Gal4), was combined with a $\mathrm{Tg}$ (UAS:tagRFP-CAAX) responder line and labeled subsets of amacrine and tectal cells (Antinucci et al., 2016). The tenm3-positive amacrine cells consistently stratified their neurites in three IPL strata but did not stratify correctly in tenm3 knockout mutants (Antinucci et al., 2016). Indeed, tenm3 expression is critical for the proper development of orientation selectivity in the retina (Antinucci et al., 2016; Antinucci and Hindges, 2018). Further studies into the classification of different teneurin positive cells, will allow us to potentially relate distinct
RGC and amacrine cell types to visual functionalities based on their morphology and stratification, with BAC transgenesis being a viable method for accomplishing this.

While the functional dimerization of teneurin into covalent, disulphide-linked homodimers mediates many of its physiological effects via homophilic interactions, all four teneurin paralogs may also participate in the formation of heterodimers (Feng et al., 2002). If multiple teneurins are expressed in a single cell type, it would be prudent to suggest that different hetero- and homodimer combinations could be utilized to establish a range of hetero- and homophilic interactions at the

TABLE 2 | Mean dendritic field diameter and area of mCitrine-labeled amacrine cells in tenm2.14-BAC-injected fish.

\begin{tabular}{|c|c|c|c|c|c|c|c|c|c|}
\hline \multirow[t]{3}{*}{ Amacrine cell morphology } & \multicolumn{6}{|c|}{ Narrow field } & \multicolumn{2}{|c|}{ Medium field } & \multirow{3}{*}{$\begin{array}{c}\text { Wide field } \\
\text { Monostratified } \\
\text { ON }\end{array}$} \\
\hline & \multicolumn{2}{|c|}{ Monostratified } & \multicolumn{4}{|c|}{ Diffuse } & \multirow{2}{*}{$\frac{\text { Monostratified }}{\text { OFF }}$} & \multirow{2}{*}{$\begin{array}{c}\text { Diffuse } \\
\text { Asym. }\end{array}$} & \\
\hline & OFF & ON & 2 bunches & Asym. & ON/OFF & ON & & & \\
\hline Dendritic field diameter ( $\mu \mathrm{m})$ & $22 \pm 2$ & $23 \pm 3$ & 13 & 24 & 16 & $12 \pm 1$ & $100 \pm 30$ & 50 & 230 \\
\hline Dendritic field area $\left(\mu \mathrm{m}^{2}\right)$ & $350 \pm 70$ & $310 \pm 50$ & 200 & 490 & 230 & $160 \pm 20$ & $1300 \pm 300$ & 1110 & 4080 \\
\hline$n$ & 8 & 5 & 1 & 1 & 1 & 2 & 2 & 1 & 1 \\
\hline
\end{tabular}

The dendritic field diameter was measured as the width of the dendritic arbor, while the dendritic field area was measured from the top views of the amacrine cells. Measurements were made with ImageJ. $n$ represents the number of cells analyzed per type. SD is shown for all cell types where $n>1$. 
cell membrane. Combined with alternative splicing events and heterophilic interactions with other cell adhesion molecules, the teneurins may form part of a wider molecular code that functions to increase the diversity of cellular interactions from a limited set of genes. In this fashion, different cell adhesion molecules that are specific for a certain cell type may interact in a combinatorial fashion to drive synaptic specificity.

Much of the focus on teneurin functionality in circuit assembly has been through studying tenm3 (Antinucci et al., 2016; Berns et al., 2018). The functional involvement of the other three paralogs, if any, is less well established and more research is needed to shed light on whether these other teneurins may also act in a similar way to regulate the precise connectivity of the nervous system during development. With genetic variations in the human teneurin gene loci implicated as a significant susceptibility factor in many neurological disabilities such as bipolar disorder (Sklar et al., 2011), intellectual disability (Tucker and Chiquet-Ehrismann, 2006) and autism spectrum disorders (Nava et al., 2012), the involvement of the teneurins in conferring precise neural circuitry is paramount. Future studies will build up on the expression data we have presented here, relating the sites of expression to teneurin functionality and synaptic connectivity. Furthering our understanding of this enigmatic process will help bring us a step closer to understanding how the intricate connections of the nervous system are established.

\section{DATA AVAILABILITY}

All datasets generated for this study are included in the manuscript and/or the Supplementary Files.

\section{REFERENCES}

Antinucci, P., and Hindges, R. (2018). Orientation-selective retinal circuits in vertebrates. Front. Neural Circuits 12:11. doi: 10.3389/fncir.2018.00011

Antinucci, P., Nikolaou, N., Meyer, M. P., and Hindges, R. (2013). Teneurin-3 specifies morphological and functional connectivity of retinal ganglion cells in the vertebrate visual system. Cell Rep. 5, 582-592. doi: 10.1016/j.celrep.2013. 09.045

Antinucci, P., Suleyman, O., Monfries, C., and Hindges, R. (2016). Neural mechanisms generating orientation selectivity in the retina. Curr. Biol. 26, 1802-1815. doi: 10.1016/j.cub.2016.05.035

Baumgartner, S., and Chiquet-Ehrismann, R. (1993). Tena, a Drosophila gene related to tenascin, shows selective transcript localization. Mech. Dev. 40, 165-176. doi: 10.1016/0925-4773(93)90074-8

Baumgartner, S., Martin, D., Hagios, C., and Chiquet-Ehrismann, R. (1994). Tenm, a Drosophila gene related to tenascin, is a new pair-rule gene. EMBO J. 13, 3728-3740. doi: 10.1002/j.1460-2075.1994.tb06682.x

Beckmann, J., Schubert, R., Chiquet-Ehrismann, R., and Müller, D. J. (2013). Deciphering teneurin domains that facilitate cellular recognition, cell-cell adhesion, and neurite outgrowth using atomic force microscopy-based single-cell force spectroscopy. Nano Lett. 13, 2937-2946. doi: 10.1021/nl401 3248

Berns, D. S., DeNardo, L. A., Pederick, D. T., and Luo, L. (2018). Teneurin3 controls topographic circuit assembly in the hippocampus. Nature 554, 328-333. doi: 10.1038/nature25463

Bibollet-Bahena, O., Okafuji, T., Hokamp, K., Tear, G., and Mitchell, K. J. (2017). A dual-strategy expression screen for candidate connectivity labels in the developing thalamus. PLoS One 12:e0177977. doi: 10.1371/journal.pone. 0177977

\section{ETHICS STATEMENT}

This work was approved by the local Animal Welfare and Ethical Review Body (King's College London), and was carried out in accordance with the Animals (Scientific Procedures) Act 1986, under license from the United Kingdom Home Office to RH.

\section{AUTHOR CONTRIBUTIONS}

$\mathrm{RH}$ designed the study. KT, MR-C, and PA carried out the experiments. KT, MR-C, PA, and AC analyzed the data. AC and $\mathrm{RH}$ wrote the manuscript with input from the other authors.

\section{FUNDING}

This work was supported by grants to RH from the Biotechnology and Biological Sciences Research Council (BB/M000664/1), the Leverhulme Trust (RPG-2017-168) and the Medical Research Council (MR/N026063/1). Costs for open access publication fees were covered by institutional block grant to King's College London.

\section{SUPPLEMENTARY MATERIAL}

The Supplementary Material for this article can be found online at: https://www.frontiersin.org/articles/10.3389/fnins. 2019.00158/full\#supplementary-material

Boucard, A. A., Maxeiner, S., and Südhof, T. C. (2014). Latrophilins function as heterophilic cell-adhesion molecules by binding to teneurins: regulation by alternative splicing. J. Biol. Chem. 289, 387-402. doi: 10.1074/jbc.M113.504779

Drabikowski, K., Trzebiatowska, A., and Chiquet-Ehrismann, R. (2005). ten-1, an essential gene for germ cell development, epidermal morphogenesis, gonad migration, and neuronal pathfinding in Caenorhabditis elegans. Dev. Biol. 282, 27-38. doi: 10.1016/j.ydbio.2005.02.017

Fascetti, N., and Baumgartner, S. (2002). Expression of Drosophila Ten-a, a dimeric receptor during embryonic development. Mech. Dev. 114, 197-200. doi: 10. 1016/S0925-4773(02)00055-2

Feng, K., Zhou, X. H., Oohashi, T., Mörgelin, M., Lustig, A., Hirakawa, S., et al. (2002). All four members of the Ten-m/Odz family of transmembrane proteins form dimers. J. Biol. Chem. 277, 26128-26135. doi: 10.1074/jbc.M203722200

Hong, W., Mosca, T. J., and Luo, L. (2012). Teneurins instruct synaptic partner matching in an olfactory map. Nature 484, 201-207. doi: 10.1038/nature10926

Jackson, V. A., Meijer, D. H., Carrasquero, M., van Bezouwen, L. S., Lowe, E. D., Kleanthous, C., et al. (2018). Structures of Teneurin adhesion receptors reveal an ancient fold for cell-cell interaction. Nat. Commun. 9, 1-9. doi: 10.1038/ s41467-018-03460-0

Kenzelmann, D., Chiquet-Ehrismann, R., Leachman, N. T., and Tucker, R. P. (2008). Teneurin-1 is expressed in interconnected regions of the developing brain and is processed in vivo. BMC Dev. Biol. 8:30. doi: 10.1186/1471-213X8-30

Leamey, C. A., Glendining, K. A., Kang, N., Wang, K. H., Sawatari, A., Tonegawa, S., et al. (2008). Differential gene expression between sensory neocortical areas: potential roles for Ten_m3 and Bcl6 in patterning visual and somatosensory pathways. Cereb. Cortex 18, 53-66. doi: 10.1093/cercor/bhm031

Leamey, C. A., Merlin, S., Lattouf, P., Sawatari, A., Zhou, X., Demel, N., et al. (2007). Ten_m3 regulates eye-specific patterning in the mammalian visual 
pathway and is required for binocular vision. PLoS Biol. 5:e241. doi: 10.1371/ journal.pbio.0050241

Leamey, C. A., and Sawatari, A. (2014). The teneurins: new players in the generation of visual topography. Semin. Cell Dev. Biol. 35, 173-179. doi: 10. 1016/j.semcdb.2014.08.007

Li, H., Bishop, K. M., and O'Leary, D. D. (2006). Potential target genes of EMX2 include Odz/Ten-M and other gene families with implications for cortical patterning. Mol. Cell Neurosci. 33, 136-149. doi: 10.1016/j.mcn.2006. 06.012

Li, J., Shalev-Benami, M., Sando, R., Jiang, X., Kibrom, A., Wang, J., et al. (2018). Structural basis for teneurin function in circuit-wiring: a toxin motif at the synapse. Cell 173, 735-748.e15. doi: 10.1016/j.cell.2018. 03.036

Mieda, M., Kikuchi, Y., Hirate, Y., Aoki, M., and Okamoto, H. (1999). Compartmentalized expression of zebrafish ten-m3 and ten-m4, homologues of the Drosophila tenm/odd Oz gene, in the central nervous system. Mech. Dev. 87, 223-227. doi: 10.1016/S0925-4773(99)00155-0

Nava, C., Lamari, F., Héron, D., Mignot, C., Rastetter, A., Keren, B., et al. (2012). Analysis of the chromosome $\mathrm{X}$ exome in patients with autism spectrum disorders identified novel candidate genes, including TMLHE. Transl. Psychiatry 2, 1-12. doi: 10.1038/tp.2012.102

Nunes, S. M., Ferralli, J., Choi, K., Brown-Luedi, M., Minet, A. D., and Chiquet-Ehrismann, R. (2005). The intracellular domain of teneurin-1 interacts with $\mathrm{MBD} 1$ and $\mathrm{CAP} /$ ponsin resulting in subcellular codistribution and translocation to the nuclear matrix. Exp. Cell Res. 305, 122-132. doi: 10.1016/j. yexcr.2004.12.020

Rubin, B. P., Tucker, R. P., Martin, D., and Chiquet-Ehrismann, R. (1999). Teneurins: a novel family of neuronal cell surface proteins in vertebrates, homologous to the Drosophila pair-rule gene product Ten-m. Dev. Biol. 216, 195-209. doi: 10.1006/dbio.1999.9503

Sklar, P., Ripke, S., Scott, L. J., Andreassen, O. A., Cichon, S., Craddock, N., et al. (2011). Large-scale genome-wide association analysis of bipolar disorder identifies a new susceptibility locus near ODZ4. Nat. Genet. 43, 977-985. doi: 10.1038/ng.943
Storey, E., Bahlo, M., Fahey, M., Sisson, O., Lueck, C. J., and Gardner, R. J. M. (2009). A new dominantly inherited pure cerebellar ataxia, SCA 30. J. Neurol. Neurosurg. Psychiatry 80, 408-411. doi: 10.1136/jnnp.2008.159459

Thisse, C., and Thisse, B. (2008). High-resolution in situ hybridization to wholemount zebrafish embryos. Nat. Protoc. 3, 59-69. doi: 10.1038/nprot.2007.514

Tucker, R. P., Beckmann, J., Leachman, N. T., Schöler, J., and ChiquetEhrismann, R. (2012). Phylogenetic analysis of the teneurins: conserved features and premetazoan ancestry. Mol. Biol. Evol. 29, 1019-1029. doi: 10.1093/molbev/ msr271

Tucker, R. P., and Chiquet-Ehrismann, R. (2006). Teneurins: a conserved family of transmembrane proteins involved in intercellular signaling during development. Dev. Biol. 290, 237-245. doi: 10.1016/j.ydbio.2005.11.038

Vysokov, N. V., Silva, J.-P., Lelianova, V. G., Ho, C., Djamgoz, M. B., Tonevitsky, A. G., et al. (2016). The mechanism of regulated release of lasso/teneurin-2. Front. Mol. Neurosci. 9:59. doi: 10.3389/fnmol.2016.00059

Young, T. R., Bourke, M., Zhou, X., Oohashi, T., Sawatari, A., Fässler, R., et al. (2013). Ten-m2 is required for the generation of binocular visual circuits. J. Neurosci. 33, 12490-12509. doi: 10.1523/JNEUROSCI.4708-12.2013

Zhou, X. H., Brandau, O., Feng, K., Oohashi, T., Ninomiya, Y., Rauch, U., et al. (2003). The murine Ten-m/Odz genes show distinct but overlapping expression patterns during development and in adult brain. Gene Expr. Patterns 3, 397405. doi: 10.1016/S1567-133X(03)00087-5

Conflict of Interest Statement: The authors declare that the research was conducted in the absence of any commercial or financial relationships that could be construed as a potential conflict of interest.

Copyright (C) 2019 Cheung, Trevers, Reyes-Corral, Antinucci and Hindges. This is an open-access article distributed under the terms of the Creative Commons Attribution License (CC BY). The use, distribution or reproduction in other forums is permitted, provided the original author(s) and the copyright owner(s) are credited and that the original publication in this journal is cited, in accordance with accepted academic practice. No use, distribution or reproduction is permitted which does not comply with these terms. 